\title{
„Die DRG-Zertifizierung zum muskuloskelettalen Radiologieexperten ist in dieser Form einzigartig!“‘
}

Wir haben mit PD Dr. med. Marc Regier, Leitender Oberarzt an der Klinik und Poliklinik für Diagnostische und Interventionelle Radiologie und Nuklearmedizin am Universitätsklinikum Hamburg-Eppendorf, über die noch junge Arbeitsgemeinschaft Bildgebende Verfahren des Bewegungsapparates gesprochen. In seiner Funktion als Mitglied des Vorstands erläutert er das Zertifizierungsprogramm der AG und berichtet über den bevorstehenden Intensivkurs Muskuloskelettale Radiologie.

DRG: Die Arbeitsgemeinschaft Bildgebende Verfahren des Bewegungsapparates wurde erst 2015 gegründet. Sie hat sich insbesondere zum Ziel gesetzt, die Zusammenarbeit von Klinik und Praxis im Bereich der Muskuloskelettalen Radiologie zu fördern und auszubauen. Ist der Graben zwischen Klinik und Praxis im Muskulo-Gebiet zurzeit so groß? Und wenn ja, wie will ihn die AG überbrücken?
Regier Die Muskuloskelettale Diagnostik ist ein sehr großes Teilgebiet der Radiologie. Das liegt einerseits an dem großen Anteil muskuloskelettaler Untersuchungen an der Gesamtdiagnostik in der täglichen Routine von Klinik und insbesondere auch Praxis - andererseits hat der Erkenntnisgewinn der letzten Jahre in der muskuloskelettalen Radiologie dazu geführt, dass die an uns herangetragenen Fragestellungen komplexer und dezidierter geworden sind. Qualitativ hochwertige Antworten sind somit nur mit entsprechender Expertise möglich. Hierfür brauchen wir einen intensiven Dialog aller muskuloskelettal interessierten Radiologen aus Klinik und Praxis unter dem Dach der Deutschen Röntgengesellschaft das ist von immenser Bedeutung.

\section{Wie wollen Sie das konkret umsetzen?}

Am ehesten im Rahmen strukturierter Fortbildungsveranstaltungen und gemeinsam erarbeiteter Untersuchungsstrategien. Und das ist eine der zentralen Aufgaben unserer Arbeitsgemeinschaft Bildgebende Verfahren des Bewegungsapparates.

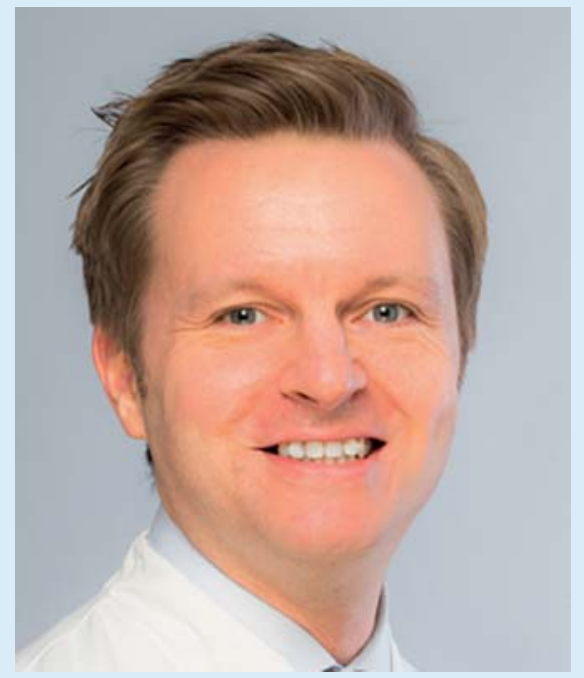

PD Dr. med. Marc Regier

Welche weiteren Ziele verfolgt die AG in den nächsten Jahren?

Um den Dialog zwischen Praxis und Klinik aufrecht zu erhalten und zu vergrößern, wollen wir möglichst viele Kolleginnen und Kollegen in die Aktivitäten der AG BVB einbinden und die Kommunikation unterei- 


\section{AG Bildgebende Verfahren des Bewegungsapparates}

in der Deutschen Röntgengesellschaft nander stärken. Praktisch heißt das: Wir bauen hierzu gerade eine interaktive Fallsammlung auf, die sich strukturell an der erfolgreich etablierten Lehr- und Lernplattform der Deutschen Röntgengesellschaft, die auf Initiative und mit großem Engagement der AG Herz- und Gefäßdiagnostik entstanden ist, orientiert. Außerdem wollen wir attraktive und innovative Fortbildungsprogramme schaffen und gleichzeitig darauf achten, dass wir neue wissenschaftliche Erkenntnisse in angemessener Weise berücksichtigen und integrieren.

Gibt es spezielle AG-Veranstaltungen, mit denen Sie Akzente setzen wollen?

Mit dem RöKo in Leipzig, der Heidelberger Summer School und dem Intensivkurs Muskuloskelettale Radiologie in Hamburg bieten wir über das Jahr verteilt Schwerpunktveran- staltungen an, die die genannten Themen vereinen und allen Interessierten die Möglichkeit bieten, ihr eigenes Wissen zu vertiefen. Alle Veranstaltungen haben übrigens eine Gemeinsamkeit: Der Besuch unterstützt den Weg zur Zertifizierung zum Muskuloskelettalen Radiologen der Deutschen Röntgengesellschaft. Dieses neuartige Zertifizierungsmodell darf als Gütesiegel der DRG verstanden werden, das den hohen Kenntnisstand des Radiologen im Schwerpunktbereich MSK ausweist und sowohl Zuweisern als auch Patienten den großen Vorteil bringt, MSK-Experten leichter auffinden zu können!

Ist das neue Zertifizierungsprogramm der AG eine komplizierte Sache?

Ganz im Gegenteil. Wir haben 2015 ein klar strukturiertes Zertifizierungsprogramm geschaffen, das sich an den Leitlinien der DRG und der Umsetzungspraxis der Deutschen Gesellschaft für Interventionelle Radiologie orientiert. Hierbei haben wir uns ganz bewusst für ein zweistufiges Modell entschieden. Die Stufe 1 als Basis-Qualifikation MSK kann bereits während der letzten zwei Jahre vor Facharztreife erreicht werden und geht über die Inhalte der Weiterbildung zum Facharzt hinaus. Daran schließt sich mit dem Spezialwissen MSK die Stufe 2 an, die den Inhaber als zertifizierten muskuloskelettalen Radiologen der Deutschen Röntgengesellschaft ausweist, der über Expertenwissen verfügt. Wie bei anderen DRG-Zertifizierungen auch müssen die hierfür notwendigen Untersuchungszahlen dokumentiert und die notwendigen Besuche ausgewählter Fortbildungsveranstaltungen bescheinigt werden. Abgeschlossen wird die Zertifizierungsstufe 2 künftig dann mit einer mündlichen Prüfung. Dieses Modell ermöglicht jedem Interessierten, der über mannigfaltiges Wissen und Erfahrung in muskuloskelettaler Radiologie verfügt, exklusiven Zugang zur Anerkennung als Experte der muskuloskelettalen Radiologie durch die Deutsche Röntgengesellschaft. Das ist in der Form einzigartig!

Vom 8. bis 9. September 2017 findet in Hamburg erstmalig der Intensivkurs Muskuloskelettale Radiologie (IMR) statt, dessen wissenschaftlicher Leiter Sie sind. Welche Schwerpunkte setzt die AG-Veranstaltung?

Das zweitägige Fortbildungsseminar in Hamburg konzentriert sich in seinem ersten Jahr auf die MRT der großen Gelenke, Erkrankungen der Wirbelsäule und die Diagnostik von Knochentumoren. Als besonderes Highlight ergänzen wir das Programm durch eine Sitzung, die aktuelle Erkenntnisse zur bildgebenden Diagnostik im Profisport vermittelt. Es wird eine ausgewogene Balance zwischen praxisrelevantem Basiswissen, Protokollempfehlungen für die tägliche Routine und innovativem Spezialwissen bieten. Die Teilnehmerinnen und Teilnehmer werden Gelegenheit haben, ihre Kenntnisse aufzufrischen und zu vertiefen. Zu guter Letzt finden am Veranstaltungswochenende die „Hamburg Cruise Days" statt, die den Fortbildungsbesuch mit Blick auf Hafen und Elbphilharmonie gesellig abrunden.

Vielen Dank für das Gespräch! 